\title{
Host Genetics and Viral Diversity: Report from a Global HIV Vaccine Enterprise Working Group
}

\author{
Andrew McMichael and Francine McCutchan on behalf of a Working Group convened by the Global HIV \\ Vaccine Enterprise.
}

\section{A. INTRODUCTION}

The Global HIV Vaccine Enterprise convened a workshop in September 2009 to discuss human and viral genetic variation and its impact on future directions for HIV vaccine research and development. The formidable challenges presented by virus and host genetic variability are interrelated and complicate vaccine development. HIV vaccine researchers need to develop innovative approaches that will facilitate addressing these questions in novel ways.

\section{B. CHALLENGES AND OPPORTUNITIES}

\section{Host Genetic Variability}

Human genetic variability influences susceptibility to HIV-1 infection, rate of disease progression and immune responses to vaccination.

Resistance to infection. Many studies have identified individuals who remain uninfected after repeated exposure to HIV-1. The clearest and the best-understood mode of protection against HIV-1 infection results from deficiency in expression of the virus co-receptor CCR5 as the result of a 32 base pair deletion in the coding sequence of the CCR5 gene ${ }^{1}$. However, only a subset of protected individuals carries the CCR5 deletion. Others appear to develop protective immune responses, elicited as the result of repeated exposure to HIV, leading to transient or locally contained infection, without becoming productively infected with the virus ${ }^{2-4}$.

HLA associations. Once infection has occurred, a number of genes are known to affect HIV-1 viral load and/or rate of disease progression with the MHC complex having the most profound influence ${ }^{5}$. Detailed HLA typing has revealed that several alleles are associated with lower viral set point or delayed progression to AIDS: ${ }^{\star} 5701$, $B^{\star} 5703, B^{\star} 5801, B^{\star} 2705$ and $B^{\star} 5101$. The mechanisms underlying this phenomenon are not fully understood, but for the B27, B51 and B57/58 families it is probable that HLA-restricted T cells play an important role by targeting conserved epitopes in the HIV-1 p24 protein ${ }^{6,7}$. Viruses can escape $\mathrm{T}$ cell recognition by mutation and subsequent selection, but this often incurs a fitness $\operatorname{cost}^{8-12}$. In contrast, rapid disease progression is associated with some subtypes of HLA B ${ }^{\star} 35$. The mechanism may involve a relatively high affinity interaction of peptide-MHC complexes with LILRB2, an HLA class I receptor on monocytes and dendritic cells, responsible for downregulation of dendritic cell function ${ }^{13}$. Genome-wide association studies (GWAS) have also identified a variant in HLA-C, the - 35 SNP that is associated with lower viral load, although its mechanism of action is not clear ${ }^{14}$. Recent GWAS studies ${ }^{15-17}$, including a first study in an African American cohort $^{18}$, have excluded the influence of many other genes previously proposed to influence the outcome of HIV-1 infection, rather than identifying further protective genes.

NK cell receptors. The killer immunoglobulin-like receptors (KIRs) are found on natural killer (NK) cells and a subset of $\mathrm{T}$ cells $\left(\mathrm{KIR}^{+} \mathrm{CD} 8^{+}\right)$. KIRs are either inhibitory or activating and have several allelic forms and gene copy number variability. They bind to class I HLA molecules focusing on the Bw4/6 region of the alpha- 1 helix. KIR3DL1 and KIR3DS1 are associated with favorable control of HIV-1 virus load or slow disease progression in individuals with the appropriate HLA Bw4 type ${ }^{19-22}$. These findings strongly implicate NK cells, and/or $\mathrm{KIR}^{+} \mathrm{CD}^{+} \mathrm{T}$ cells, in the control of $\mathrm{HV}-1$ infection.

The importance of host genetics in vaccine studies may also be significant. Host genetic variation might influence response to vaccination and thereby confound vaccine efficacy studies. This issue may become especially important in vaccine trials involving genetically diverse populations where there may be as yet undiscovered genetic variation that could affect clinical outcome.

\section{HIV Variability and its Sources}

HIV-1 is one of the most genetically variable viruses known. Variation is fueled by an error-prone reverse transcriptase and magnified by frequent recombination events. From the time it entered the human population, HIV has diversified into multiple genetic subtypes, or clades, each with a distinct geographic distribution. Moreover, some inter-subtype recombinant forms of the virus have gained widespread prevalence and are therefore important targets of HIV vaccine devel-

Andrew McMichael is at the Weatherall Institute, Oxford, United Kingdom; Francine McCutchan is at the Bill \& Melinda Gates Foundation, Seattle, United States. Other members of the Enterprise Working Group included: Mary Carrington, National Cancer Institute, USA; John Coffin, National Cancer Institute \& Tufts University, USA; Mark Connors, National Institute of Allergy and Infectious Diseases, USA; Josephine Cox, International AIDS Vaccine Initiative, USA; Mark Feinberg, Merck \& Co. Inc., USA; Philip Goulder, University of Oxford, UK; Beatrice Hahn, University of Alabama at Birmingham, USA; Edward Karamov, Institute of Immunology \& Ivanovsky Institute of Virology, Russia; Bette Korber, Los Alamos National Laboratory, USA; Norman Letvin, Harvard University, USA; Angela McLean, University of Oxford, UK; Lynn Morris, National Institute for Communicable Diseases, South Africa; Saladin Osmanov, World Health Organization, Switzerland; Francis Plummer, University of Manitoba, Canada; Sarah Rowland-Jones, University of Oxford, UK; Sara Sawyer, University of Texas at Austin, USA; Gabriella Scarlatti, San Raffaele Scientific Institute, Italy; Stuart Shapiro, National Institute of Allergy and Infectious Diseases, USA; George Shaw, University of Alabama at Birmingham, USA; Amalio Telenti, University of Lausanne, Switzerland; loannis Theodorou, Hôpital Pitié Salpêtrière, France; Bruce Walker, Ragon Institute, USA; Carolyn Williamson, University of Cape Town, South Africa; Otto Yang, University of California, Los Angeles, USA. 
opment. Nine genetic subtypes (A,B,C,D,F,G,H,J,K) circulate in the epidemic and two recombinant forms (CRF01_AE and CRF02_AG) are also of major importance. Many other recombinant forms circulate at lower levels or in a more limited geographic range. The seemingly limitless capacity for tolerating genetic variation makes HIV one of the most challenging targets for coverage by vaccines (reviewed $\mathrm{in}^{23}$ ). The current focus of vaccine development is on a handful of clades or recombinant forms that infect large populations or wide geographic areas; in order of global distribution, these are clade C, CRF02_AG, clades A, B, D, and CRF01_AE.

Antibodies and variability. The envelope gp120 protein is the most variable part of the virus ( $>10 \%$ amino acid intraclade differences and $>20 \%$ interclade differences). The five regions of hypervariability (V1V5) are under strong selective pressure by neutralizing antibodies, from which the virus rapidly escapes ${ }^{24}$. More conserved regions of the envelope are hidden from antibodies in the internal faces of the trimer, masked by glycosylation, or are conformationally labile. The membrane-proximal gp41 is less variable and contains sites that are conserved and recognized by neutralizing monoclonal antibodies with broad specificity (bNAbs) ${ }^{25}$. However, membrane lipid has an influence on binding of this type of antibody and, if targeted by a vaccine, may lead to autoreactivity with lipid antigens ${ }^{25,26}$.

An extensive search and analysis of HIV-1 envelope-specific monoclonal antibodies has led to isolation of novel bNAbs ${ }^{27}$. Unfortunately, it has not yet been possible to efficiently trigger this type of antibody through vaccination ${ }^{28}$, because the epitopes recognized by the bNAbs characterized to date are rarely seen by antibodies in vaccinated individuals. Nevertheless, the isolation of bNAbs has important implications for vaccine design ${ }^{29}$ - development of immunogens able to elicit bNAbs at high titer represents an actively pursued strategy to overcome HIV sequence and epitope variability.

T cell responses and virus variability. CD8+ T cell receptors are highly sensitive to amino acid changes in the targeted epitope: epitopes with single mutations are recognized by only a minority of $\mathrm{T}$ cell clones and double mutants are only rarely recognized ${ }^{30,31}$. The rapid selection of virus escape mutations by CD8 + T cells in acute HIV infection dramatically illustrates the ease with which HIV can escape these responses ${ }^{32}$. Thus, trials that test candidate vaccines based on clades that differ to the ones that the trial population is exposed to are likely to fail.

The impact of HIV variability on vaccine design is manifest in two ways. First, the vaccine and the virus may mismatch, even within the same clade, to the extent that the vaccine-induced responses are ineffective. This factor may have contributed to the failure of the Merck Step trial vaccine ${ }^{33}$, which only elicited $\mathrm{T}$ cell responses to a median of three epitopes, of which only one was in gag, the most 'protective' protein in CTL recognition studies ${ }^{34}$. Second, vaccine-elicited responses may be easily evaded if the response focuses on a variable region of the virus. For development of a broadly efficacious vaccine, it will be important to elicit responses of considerable breadth, unlike the narrow responses that occur during acute natural infection and result in a cycle of selection and escape.

To overcome these problems, immune responses could be stimulated to all the common variants (for example, by using mosaic vaccines ${ }^{35}$ ), or to the most conserved regions of the virus proteome $\mathrm{e}^{36}$. It is clear from the above considerations that HIV variability and its ability to escape both the humoral and $\mathrm{T}$ cell responses remain unresolved challenges. A better understanding of the fitness costs of escape, and the role of viral compensatory mutations, are therefore high priorities ${ }^{6,9,37}$.

Virus variability and innate immune responses. Not all selected mutations observed in acute HIV-1 infection can be explained by $\mathrm{T}$ cell escape, antibody escape or reversion to consensus (presumably
Table 1. Summary of priorities and recommendations

Priority 1. Create enabling environments that foster innovative, collaborative, and global approaches

- Create new and support existing "centers of excellence" to foster diverse intra- and interdisciplinary collaborations on a global scale.

- Collect and share specimens and data on global host and viral diversity.

Priority 2: Understanding the role of host genetics in HIV vaccine research

- Ensure that the contributions of the host genome to variability in acquisition and disease phenotypes are considered in clinical trials and population cohorts.

- Complement human genetic studies with parallel investigation in NHP models.

Priority 3: Understand viral evolution, and exploit this information in vaccine development

- Establish, expand and coordinate large multidisciplinary efforts to study infected individuals during the earliest stage after infection through the first 1-2 years.

- Use NHP models to dissect the earliest events after infection that are currently not accessible for studies in humans.

- Develop a multi-pronged approach, using a diversity of models and assays, to study relative impact of selective forces on viral evolution and constraints on viral genetic flexibility.

Priority 4: Defining how the interplay between viral and host diversity contributes to immune containment of the virus

- Develop novel assays to measure antiviral activity responsible for immune containment.

- Initiate population studies that address viral diversity in the context of host genetic environment.

- Prioritize and facilitate phase I trials that address host and virus diversity.

to increase fitness), suggesting that selection by the innate immune response also contributes to viral variation. Innate immune responses are the first to appear in acute HIV-1 infection, leading to activation and expansion of NK cells, dendritic cells and macrophages. The specific role of the innate immune response in viral containment in vivo remains poorly understood.

\section{WAY FORWARD}

The Working Group identified several priority areas that remain critical challenges on the path to a safe and effective HIV vaccine.

\section{Priority 1: Create enabling environments that foster innovative,} collaborative, and global approaches

Recent advances in sequencing technology provide an unprecedented opportunity for developing a detailed picture of both human and viral diversity globally and in individual patients. The speed and utility of these studies rely on rapid sharing of both materials and data. Global collaboration is essential for addressing questions on this global scale. In addition, it requires new talent to develop the tools and technology necessary for the corresponding sophisticated data deposition and analysis. Thus, an enabling environment is necessary to foster collaboration and attract new talent to the field. Such an environment will include intra- and interdisciplinary collaborations, an integrated global network for population studies and agreed-upon mechanisms to share specimens and data from anywhere in the world.

\section{Recommendations}

Create new and support existing "centers of excellence" to foster diverse intra- and interdisciplinary collaborations on a global scale. Interdisciplinary centers should be supported to attract scientists from other areas of immunology, from cell and molecular biology, and from physical sciences and mathematics. New talent and resources may be 
found in emerging economies, like China and India, and in regions hardest hit by the HIV-1 epidemic. This talent should be engaged in international efforts through collaborations with existing centers in the developing world and nurtured by training and mentoring for young investigators. Strong scientific leadership in these centers is critical to address key questions in HIV vaccine research and development. Collaborations between clinicians, epidemiologists, local medical personnel, and laboratory scientists need to be well-directed and equally valued.

Collect and share specimens and data on global host and viral diversity. Besides recruiting new talent, an enabling environment requires broad access to reagents, high-quality specimens and data from population studies and unique cohorts. A major limitation in study of host and virus diversity is access to representative populations and adequate samples. There is a need for such studies in populations outside those infected with clade $\mathrm{B}$ and $\mathrm{C}$ viruses. Creation of a database of cohort studies worldwide, together with standardized protocols and procedures, will be needed.

\section{Priority 2: Understanding the role of host genetics in HIV vaccine research}

New high-throughput genetic approaches have the potential to identify major genetic factors contributing to clinical outcome in HIV-1 infection. Ideally, every human gene that impacts on each mode of HIV transmission and disease outcome should be identified to improve our understanding of the mechanisms of protection. To accomplish this goal, investigated populations should include geographically-diverse regions and extreme phenotype cohorts, such as elite controllers, rapid progressors and exposed uninfected individuals.

Non-human primates (NHP) are valuable in defining the early events following mucosal infection, and testing novel vaccine concepts. Thus, the genetic structure of NHP and its impact on vaccination and infection needs further investigation.

\section{Recommendations}

Ensure that the contributions of the host genome to variability in acquisition and disease phenotypes are considered in clinical trials and population cohorts. Complete genetic characterization of infected trial participants (vaccinated and placeboes) is important for understanding the impact of host diversity on vaccine responses and the interplay between the genetics of the host and the virus. Epidemiological studies should use advanced statistical methods and sophisticated study designs that take into account genetics of the studied population.

Complement human genetic studies with parallel investigation in NHP models. The key role of the NHP model in HIV vaccine research makes it imperative to understand the contribution of genetics to vaccine-induced protective immune responses and the outcome of SIV infection.

\section{Priority 3: Understand viral evolution, and exploit this information in vaccine development}

Understanding the selective forces acting on viral populations and constrains on viral immune escape is central to developing an HIV-1 vaccine. Efforts in this area should go beyond measurements of immune pressure by CTL and neutralizing antibodies to consider the effects of non-neutralizing antibodies, impact of diversity of target cells, contribution of long-lived latent pools, role of genetic drift, and many other potentially important factors. These analyses will require in vivo, in vitro and in silico models of viral replication, including mathematical modeling that takes into account structural and functional penalties of virus mutation. In particular, better assays for measuring viral fitness are needed to reflect the environment in which the virus replicates in vivo.

The dynamics of viral sequence changes in natural infection suggest the immune response exerts its maximum impact on the virus during acute infection. The nature of the immune responses and the level of viral control and diversification at this stage likely determine the ultimate disease course. Current studies of acute infection have explored the transmission bottleneck and early immune responses (reviewed in ${ }^{38}$ ). These studies, with strong bioinformatics support, should be expanded to include larger numbers of patients from genetically-diverse populations with different contributing risk factors. Coordination through protocol and data sharing and the use of common analytical tools will be vital.

While detailed studies on breakthrough infections in human participants in future HIV vaccine trials are invaluable, NHPs present the most convenient model to study how vaccine-induced responses influence the course of events early after infection. Studies in macaques challenged with SIV are crucial to understand early mucosal events, the mechanisms determining the bottleneck of the founder virus and the role of very early innate immune responses.

\section{Recommendations}

Establish, expand and coordinate large multidisciplinary efforts to study infected individuals during the earliest stage after infection through the first 1-2 years. Whenever possible, these studies should include vaccine recipients who acquire HIV-1 infection.

Use NHP models to dissect the earliest events after infection that are currently not accessible for studies in humans. Use the NHP model to study viral diversification and to define drivers of viral evolution.

Develop a multi-pronged approach, using a diversity of models and assays, to study relative impact of selective forces on viral evolution and constraints on viral genetic flexibility. This approach should rely on accelerated efforts to develop and deploy new viral fitness assays and more complex mathematical models of virus evolution.

\section{Priority 4: Defining how the interplay between viral and host diversity contributes to immune containment of the virus}

How does the host genome influence the ability of immune responses to effectively deal with virus diversity? Half of all elite controllers carry the HLA B57 allele ${ }^{39}$, but not all B57 infected patients are elite controllers. We need to understand the mechanisms by which advantageous HLA types confer protection against the virus and whether this protection can be activated with vaccines in genetically diverse populations. Progress in this area requires answers to two key questions. First, we need to understand the competing influences for the virus between escape from host immune responses and the associated fitness costs. Second, the mechanisms responsible for immunodominance of T- and B-cell responses are not understood.

These questions can be answered in exploratory phase I studies that take into account both host and virus diversity. These studies should explore strategies to increase the strength, the breadth (number of epitopes) and the specific targeting of responses (immunodominance).

\section{Recommendations}

Develop novel assays to measure antiviral activity responsible for immune containment.

Initiate population studies that address viral diversity in the context of host genetic environment.

Prioritize and facilitate phase I trials that address host and virus diversity. 


\section{CONCLUSIONS}

Host genetic diversity has major influences on the outcomes of both HIV-1 infection and host responses to infection. Vaccine strategies need to take this genetic diversity into account. Viral diversity needs to be understood in the context of all the selective forces acting on the virus at different stages of infection. Rational development of an effective vaccine will need to embrace all these issues.

1. Samson, M. et al. Resistance to HIV-1 infection in caucasian individuals bearing mutant alleles of the CCR-5 chemokine receptor gene. Nature 382, 722-725 (1996).

2. Rowland-Jones, S. et al. HIV-specific cytotoxic T-cells in HIV-exposed but uninfected Gambian women. Nat. Med. 1, 59-64 (1995).

3. Stranford, S.A. et al. Lack of infection in HIV-exposed individuals is associated with a strong CD8(+) cell noncytotoxic anti-HIV response. Proc. Natl. Acad. Sci. USA 96, 1030-1035 (1999).

4. Zhu, T. et al. Persistence of extraordinarily low levels of genetically homogeneous human immunodeficiency virus type 1 in exposed seronegative individuals. J. Virol. 77, 6108-6116 (2003).

5. Fellay, J. et al. A whole-genome association study of major determinants for host control of HIV-1. Science 317, 944-947 (2007).

6. Schneidewind, A. et al. Structural and functional constraints limit options for cytotoxic T-lymphocyte escape in the immunodominant HLA-B27-restricted epitope in human immunodeficiency virus type 1 capsid. J. Virol. 82, 5594-5605 (2008).

7. Frater, A.J. et al. Effective T-cell responses select human immunodeficiency virus mutants and slow disease progression. J. Virol. 81, 6742-6751 (2007).

8. Leslie, A. et al. Transmission and accumulation of CTL escape variants drive negative associations between HIV polymorphisms and HLA. J. Exp. Med. 201, 891-902 (2005).

9. Leslie, A.J. et al. HIV evolution: CTL escape mutation and reversion after transmission. Nat. Med. 10, 282-289 (2004).

10. Martinez-Picado, J. et al. Fitness cost of escape mutations in p24 Gag in association with control of human immunodeficiency virus type 1. J. Virol. 80, 3617-3623 (2006).

11. Kawashima, Y. et al. Adaptation of HIV-1 to human leukocyte antigen class I. Nature 458, 641-645 (2009).

12. Schneidewind, A. et al. Escape from the dominant HLA-B27-restricted cytotoxic T-lymphocyte response in Gag is associated with a dramatic reduction in human immunodeficiency virus type 1 replication. J. Virol. 81, 12382-12393 (2007).

13. Huang, J. et al. HLA-B*35-Px-mediated acceleration of HIV-1 infection by increased inhibitory immunoregulatory impulses. J. Exp. Med. 206, 2959-2966 (2009).

14. Thomas, R. et al. HLA-C cell surface expression and control of HIV/AIDS correlate with a variant upstream of HLA-C. Nat. Genet. 41, 1290-1294 (2009).

15. Le Clerc, S. et al. Genomewide association study of a rapid progression cohort identifies new susceptibility alleles for AIDS (ANRS Genomewide Association Study 03). J. Infect. Dis. 200, 1194-1201 (2009).

16. Limou, S. et al. Genomewide association study of an AIDS-nonprogression cohort emphasizes the role played by HLA genes (ANRS Genomewide Association Study 02). J. Infect. Dis. 199, 419-426 (2009).

17. Fellay, J. et al. Common genetic variation and the control of HIV-1 in humans. PLoS Genet. 5, e1000791 (2009)
18. Pelak, $\mathrm{K}$ et al. Host determinants of HIV-1 control in African Americans. J. Infect Dis. 201, 1141-1149 (2010).

19. Martin, M.P. et al. Epistatic interaction between KIR3DS1 and HLA-B delays the progression to AIDS. Nat. Genet. 31, 429-434 (2002).

20. Martin, M.P. et al. Innate partnership of HLA-B and KIR3DL1 subtypes against HIV-1. Nat. Genet. 39, 733-740 (2007)

21. Alter, G. et al. Differential natural killer cell-mediated inhibition of HIV-1 replication based on distinct KIR/HLA subtypes. J. Exp. Med. 204, 3027-3036 (2007).

22. Alter, G. et al. HLA class I subtype-dependent expansion of KIR3DS1+ and KIR3DL1+ NK cells during acute human immunodeficiency virus type 1 infection. J. Virol. 83 6798-6805 (2009)

23. Anastassopoulou, C.G. \& Kostrikis, L.G. Global genetic variation of HIV-1 infection Curr. HIV Res. 4, 365-373 (2006).

24. Moore, P.L., Gray, E.S. \& Morris, L. Specificity of the autologous neutralizing antibody response. Curr. Opin. HIV AIDS 4, 358-363 (2009).

25. Zwick, M.B. et al. Anti-human immunodeficiency virus type 1 (HIV-1) antibodies $2 \mathrm{~F} 5$ and $4 \mathrm{E} 10$ require surprisingly few crucial residues in the membrane-proximal external region of glycoprotein gp41 to neutralize HIV-1. J. Virol. 79, 1252-1261 (2005).

26. Scherer, E.M., Zwick, M.B., Teyton, L. \& Burton, D.R. Difficulties in eliciting broadly neutralizing anti-HIV antibodies are not explained by cardiolipin autoreactivity. AIDS 21, 2131-2139 (2007)

27. Walker, L.M. et al. Broad and potent neutralizing antibodies from an African donor reveal a new HIV-1 vaccine target. Science 326, 285-289 (2009).

28. Karlsson Hedestam, G.B. et al. The challenges of eliciting neutralizing antibodies to HIV-1 and to influenza virus. Nat. Rev. Microbiol. 6, 143-155 (2008).

29. Mascola, J.R. \& Montefiori, D.C. The role of antibodies in HIV vaccines. Annu. Rev. Immunol. 28, 413-444 (2010).

30. Burrows, S.R., Rodda, S.J., Suhrbier, A., Geysen, H.M. \& Moss, D.J. The specificity of recognition of a cytotoxic T Iymphocyte epitope. Eur. J. Immunol. 22, 191-195 (1992).

31. Lee, J.K. et al. T cell cross-reactivity and conformational changes during TCR engagement. J. Exp. Med. 200, 1455-1466 (2004).

32. Goonetilleke, N. et al. The first T cell response to transmitted/founder virus contributes to the control of acute viremia in HIV-1 infection. J. Exp. Med. 206, 1253-1272 (2009).

33. Buchbinder, S.P. et al. Efficacy assessment of a cell-mediated immunity HIV-1 vaccine (the Step Study): a double-blind, randomised, placebo-controlled, test-of-concept trial. Lancet 372, 1881-1893 (2008).

34. Kiepiela, P. et al. CD8+ T-cell responses to different HIV proteins have discordan associations with viral load. Nat. Med. 13, 46-53 (2007).

35. Fischer, W. et al. Polyvalent vaccines for optimal coverage of potential T-cell epitopes in global HIV-1 variants. Nat. Med. 13, 100-106 (2007).

36. Létourneau, S. et al. Design and pre-clinical evaluation of a universal HIV-1 vaccine. PLOS ONE 2, e984 (2007).

37. Kelleher, A.D. et al. Clustered mutations in HIV-1 gag are consistently required for escape from HLA-B27-restricted cytotoxic T lymphocyte responses. J. Exp. Med. 193 375-386 (2001).

38. McMichael, A.J., Borrow, P., Tomaras, G.D., Goonetilleke, N. \& Haynes, B.F. The immune response during acute HIV-1 infection: clues for vaccine development. Nat. Rev. Immunol. 10, 11-23 (2010).

39. Pereyra, F. et al. Genetic and immunologic heterogeneity among persons who control HIV infection in the absence of therapy. J. Infect. Dis. 197, 563-571 (2008). 